\title{
The Effects of Patchy Reionization on Satellite Galaxies of the Milky Way
}

\section{Citation}

Lunnan, Ragnhild, Mark Vogelsberger, Anna Frebel, Lars Hernquist, Adam Lidz, and Michael Boylan-Kolchin. 2012. "THE EFFECTS OF PATCHY REIONIZATION ON SATELLITE GALAXIES OF THE MILKY WAY." The Astrophysical Journal 746 (1): 109. https:// doi.org/10.1088/0004-637x/746/1/109.

\section{Permanent link}

http://nrs.harvard.edu/urn-3:HUL.InstRepos:41381646

\section{Terms of Use}

This article was downloaded from Harvard University's DASH repository, and is made available under the terms and conditions applicable to Open Access Policy Articles, as set forth at http:// nrs.harvard.edu/urn-3:HUL.InstRepos:dash.current.terms-of-use\#OAP

\section{Share Your Story}

The Harvard community has made this article openly available. Please share how this access benefits you. Submit a story.

Accessibility 
APJ IN PRESS

Preprint typeset using $\mathrm{LAT}_{\mathrm{E}} \mathrm{X}$ style emulateapj v. 5/2/11

\title{
THE EFFECTS OF PATCHY REIONIZATION ON SATELLITE GALAXIES OF THE MILKY WAY
}

\author{
Ragnhild Lunnan $^{1}$, Mark Vogelsberger ${ }^{1}$, Anna Frebel ${ }^{1}$, Lars Hernquist ${ }^{1}$, Adam Lidz ${ }^{2}$ and Michael \\ BOYLAN-KOLCHIN ${ }^{3}$ \\ ${ }^{1}$ Harvard-Smithsonian Center for Astrophysics, 60 Garden Street, Cambridge, MA 02138 \\ 2 Department of Physics \& Astronomy, University of Pennsylvania, 209 South 33rd Street, Philadelphia, PA 19104 and \\ 3 Center for Galaxy Evolution, 4129 Reines Hall, University of California, Irvine, CA 92697 \\ ApJ in press
}

\begin{abstract}
We combine the high-resolution Aquarius simulations with three-dimensional models of reionization based on the initial density field of the Aquarius parent simulation, Millennium-II, to study the impact of patchy reionization on the faint satellite population of Milky Way halos. Because the Aquarius suite consists of zoom-in simulations of halos in the Millennium-II volume, we follow the formation of substructure and the growth of reionization bubbles due to the larger environment simultaneously, and thereby determine the reionization redshifts of satellite candidates. We do this for four different reionization models, and also compare results to instantaneous reionization. Using a simple procedure for selecting satellites and assigning luminosities in the simulations, we compare the resulting satellite populations. We find that the overall number of satellites depends sensitively on the reionization model, with a factor of 3-4 variation between the four models for a given host halo, although the difference is entirely in the population of faint satellites $\left(M_{V}>-10\right)$. In addition, we find that for a given reionization model the total number of satellites differs by $10-20 \%$ between the patchy and homogeneous scenarios, provided that the redshift is chosen appropriately for the instantaneous case. However, the halo-halo scatter from the six Aquarius halos is large, up to a factor of 2-3, and so is comparable to the difference between reionization scenarios. In order to use the population of faint dwarf galaxies around the Milky Way as a probe of the local reionization history, then, it is necessary to first better understand the general distribution of substructure around Milky Way-mass halos.
\end{abstract}

Subject headings: cosmology: theory - galaxies: dwarf - early universe - methods: numerical

\section{INTRODUCTION}

A major challenge to the $\Lambda \mathrm{CDM}$ cosmological model is its over-prediction of substructure on small scales compared to the actual observed number of dwarf galaxies in the Local Group (the so-called "missing satellite problem"; Moore et al. 1999; Klypin et al. 1999). The most well-studied explanation for this discrepancy is that the lowest mass halos were inefficient at forming stars, so that most of these substructures remain dark. Indeed, several processes can suppress star formation and reduce the number of visible halos, including tidal stripping of satellites (e.g., Kravtsov et al. 2004), supernova feedback, and the increase of gas temperature due to cosmic reionization (Efstathiou 1992; Gnedin 2000; Benson et al. 2002a; Somerville 2002; Madau et al. 2008). Dynamical interactions between dwarfs (D'Onghia et al. 2009) or between dwarfs and the luminous disks of large halos (D'Onghia et al. 2010) may also play a role.

The discovery of a population of "ultra-faint" dwarf galaxies (UFDs; $L<10^{5} L_{\odot}$ ) in the Sloan Digital Sky Survey (SDSS) (e.g., Willman et al. 2005; Belokurov et al. 2006; Zucker et al. 2006) has roughly doubled the number of known satellites, alleviating the problem somewhat. Based on the sky coverage and sensitivity of SDSS, estimates of a completeness-corrected luminosity function down to $M_{V} \sim-2$ (Koposov et al. 2008; Tollerud et al. 2008) are available. The possible number of faint satellites could be up to the order of hun-

rlunnan@cfa.harvard.edu dreds, but due to the incomplete sky coverage of SDSS, the exact number remains uncertain.

The discovery of these low-luminosity dwarfs has also raised many new questions, however. Follow-up observations have shown that they are the faintest, most dark matter-dominated and metal-poor systems known (Simon \& Geha 2007; Kirby et al. 2008), with metallicity patterns similar to the lowest metallicity stars in the halo (e.g., Frebel et al. 2010). In order to understand the cosmological context of UFDs, including their relationship to the first galaxies, and to the building blocks of the stellar halo, it is important to be able to model conditions in the universe at the time of their formation (e.g., Frebel \& Bromm 2010).

Semi-analytic models of galaxy formation have been able to explain the observed number of satellites by invoking various feedback mechanisms, including an external reionization field (e.g., Benson et al. 2002b; Macciò et al. 2010; Li et al. 2010). According to this scenario, as the intergalactic medium is heated by reionization, gas can no longer be accreted by and cool within halos below a certain virial temperature, inhibiting further star formation in the lowest mass halos. However, these models generally rely on a simplified, uniform reionization field, which is likely quite different from reality. Simulations of reionization indicate that it was likely extended in time and spatially inhomogeneous, with a wide range in reionization redshifts for different parts of the universe (Barkana \& Loeb 2004; Lidz et al. 2007; McQuinn et al. 2007; Ahn et al. 2009; Alvarez et al. 2009). 
Several recent studies have examined various aspects of inhomogeneous (patchy) reionization and its consequences for galaxy formation. Muñoz et al. (2009) combined a reionization model with merger trees drawn from the Via Lactea II simulation, and used the observed properties of satellites to constrain model parameters. Alvarez et al. (2009) combined N-body and threedimensional reionization calculations to investigate the relationship between reionization history and local environment. Busha et al. (2010) used their resulting distribution of reionization redshifts together with the Via Lactea II subhalos, and found that the resulting number of Milky Way satellites can vary by an order of magnitude. Understanding the connection between environment, reionization epoch, and faint satellites, then, can in principle help us to understand how the Milky Way formed.

Our work is similar in spirit to the above efforts, in that we aim to examine how the history of reionization impacts the abundance of faint satellites around Milky Way-like halos. However, our methods differ from the earlier studies in that we combine reionization calculations with high resolution zoom-in simulations of Milky Way-sized halos in the same box, enabling us to track the growth of reionization bubbles and dark matter substructure simultaneously. This means that, subject to the approximations inherent to our treatment, the reionization history is completely determined by the large-scale environment, and infalling subhalos can have different reionization redshifts than the Milky Way-like final host. Moreover, since we have six high resolution halos available, we can roughly estimate the consequences of cosmic variance, rather than assuming that a single dark matter halo is representative of the Milky Way. Comparing the results from the patchy scenarios with instantaneous and homogeneous reionization models, we aim to quantify how much of a difference ignoring the patchiness of reionization makes, and to what extent the faint satellites trace the local reionization epoch.

This paper is organized as follows: Section 2 describes the simulations that are the basis of our analysis: the Aquarius suite of Milky Way halos, and reionization calculations based on its "parent" simulation, Millennium II. Our approach is described in Section 3 detailing how we pick out satellite candidates from the merger trees, and assign luminosities to them. Section 4 describes our results, showing luminosity functions in different scenarios, and exploring the differences between the patchy and homogeneous models. Our conclusions are summarized in Section 5 .

\section{SIMULATION DATA}

\subsection{The Aquarius simulations}

Our modeling is based on the Aquariug simulations (Springel et al. 2008), a suite of six highly resolved Milky Way sized dark matter halos. These are re-simulations of six halos from a lower resolution version $\left(900^{3}\right.$ particles) of the Millennium-II simulation, which is a periodic box of size $100 h^{-1} \mathrm{Mpc}$ containing $2160^{3}$ particles (Boylan-Kolchin et al. 2009). The six halos were selected on the basis of final mass, and by not having a massive

\footnotetext{
${ }^{1}$ http://www.mpa-garching.mpg.de/aquarius/
}

Table 1

Basic parameters of the six Aquarius halos.

\begin{tabular}{cccc}
\hline \hline Name & $m_{p}\left(M_{\odot}\right)$ & $M_{200}\left(M_{\odot}\right)$ & $r_{200}(\mathrm{kpc})$ \\
\hline Aq-A-2 & $1.370 \times 10^{4}$ & $1.842 \times 10^{12}$ & 245.88 \\
Aq-B-2 & $6.447 \times 10^{3}$ & $8.194 \times 10^{11}$ & 187.70 \\
Aq-C-2 & $1.399 \times 10^{4}$ & $1.774 \times 10^{12}$ & 242.82 \\
Aq-D-2 & $1.397 \times 10^{4}$ & $1.774 \times 10^{12}$ & 242.85 \\
Aq-E-2 & $9.593 \times 10^{3}$ & $1.185 \times 10^{12}$ & 212.28 \\
Aq-F-2 & $6.776 \times 10^{3}$ & $1.135 \times 10^{12}$ & 209.21 \\
\hline
\end{tabular}

close neighbor at $z=0$ (no late-time major merger); otherwise they were selected randomly. We note that one of these halos (the F halo) is somewhat of an outlier in terms of its merger history, in that it experienced its last major merger at $z=0.7$.

The same cosmological parameters were adopted as for the original Millennium simulation (Springel et al. 2005): $\Omega_{m}=0.25, \Omega_{\Lambda}=0.75, \sigma_{8}=0.9, n_{s}=1$ and Hubble constant $H_{0}=100 h \mathrm{~km} \mathrm{~s}^{-1} \mathrm{Mpc}^{-1}=73 \mathrm{~km} \mathrm{~s}^{-1}$ $\mathrm{Mpc}^{-1}$. While these are consistent within the uncertainties with the parameters estimated from the three-year Wilkinson Microwave Anisotropy Probe (WMAP) data (Spergel et al. 2007), the value of $\sigma_{8}=0.9$ is slightly higher than that inferred from the most recent measurements (Komatsu et al. 2011). In principle, the adopted $\sigma_{8}$ could lead to an overestimate of the number of halos collapsing around the reionization epoch, but studies like Bovlan-Kolchin et al. (2011a) suggest this would have a relatively minor effect.

All six halos (labeled 'A' to ' $\mathrm{F}$ ') were simulated with at least two different resolutions; we use the higher of the two ("level 2"; softening length of $65 \mathrm{pc}$ ) in our analysis. Table1 1ists the particle mass, final halo mass, and virial radius of each of the halos. Mass and radius are listed as $M_{200}$ and $r_{200}$, defined as the mass enclosed in a sphere with mean density 200 times the critical density, and the corresponding virial radius.

Snapshots are stored at 128 output times, from redshift 127 to 0 , equally spaced in $\log a$ where $a=1 /(1+z)$. For each snapshot, subhalos are identified using the SUBFIND algorithm (Springel et al. 2001), and linked between snapshots in a merger tree. Since SUBFIND requires a minimum of 20 gravitationally bound particles to define a subhalo, the smallest halo masses we resolve are of order a few times $10^{5}$ solar masses. The merger trees are our starting point for identifying subhalos in the simulation that may host present-day luminous satellite galaxies.

\subsection{Reionization calculations}

The Aquarius halos are drawn from a larger parent simulation, so we can use the parent box to calculate how regions around these halos reionize depending on their environment. We use the semi-analytic approximation of Zahn et al. (2007), based on the excursion set treatment of Furlanetto et al. (2004b,a). This semi-analytic model assumes that galaxies reside in halos above some minimum mass, $M_{\min }$, and that the number of ionizing photons produced by a galaxy is directly proportional to its host halo mass. A given region is considered ionized when the collapse fraction - i.e., the fraction of matter that lies in halos above the minimum mass - in the region exceeds some threshold value. This threshold de- 


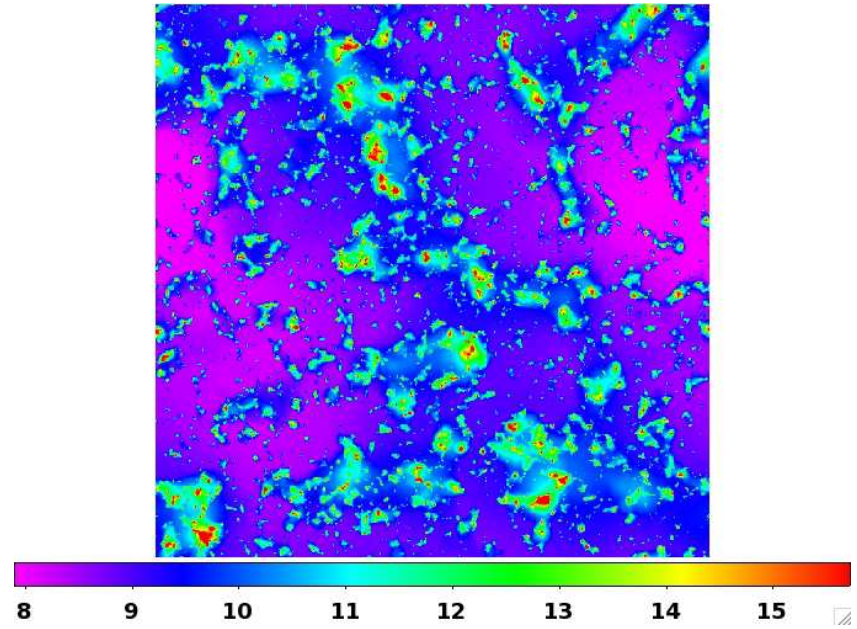

Figure 1. Slice through a reionization map from Model 1, illustrating the patchy nature of the process. The color indicates the redshift of reionization. The slice is $100 h^{-1} \mathrm{Mpc}$ on a side, and $195 h^{-1} \mathrm{kpc}$ (the cell size in the reionization calculations) thick. pends on the efficiency of the ionizing sources, and is smaller for models with more efficient sources. For the purpose of modeling reionization, we fix the minimum mass at $M_{\min }=10^{8} M_{\odot}$, and consider several models for the source efficiencies, spanning a range of possibilities for the timing and duration of reionization.

We take the initial conditions of the Millennium-II simulation as input to generate reionization maps for the cube of $100 h^{-1} \mathrm{Mpc}$ per side. Reionization redshifts are tagged spatially, at a resolution of 512 cells per side, or about $195 h^{-1} \mathrm{kpc}$. As an example, a slice through one of the reionization cubes is shown in Fig. 1, illustrating the patchiness of the process 2

We calculate four different models, including a very efficient reionization that completes around redshift 12 , and three gradually more extended models. The distributions of reionization redshifts in each model, over the cells in the box, are summarized in Table 2 and Fig. 2, In the Table, $\left\langle z_{\text {reion }}>\right.$ and $\sigma_{z}$ refer to the mean and standard deviation of the reionization redshifts across the cells in the box, and $z_{\text {end }}$ is the redshift where the process is complete. The quantity $\tau_{e}$ is the Thomson optical depth; the 7-year WMAP value for this is $\tau_{e}=0.088 \pm 0.015$ (Komatsu et al. 2011).

With these maps, we can then find the reionization redshift of any given subhalo as follows. At each snapshot, we locate the position of the subhalo in the parent box, and look up the redshift at which this region of the parent box reionizes. The first snapshot where this redshift is earlier than the redshift of the snapshot, flags the subhalo as having entered a reionized region, and we define this as the reionization redshift of the subhalo. Note that this allows not only for different reionization redshifts for the six main Aquarius halos, but also that their subhalos ending up as satellites are not required to reionize at the same time as the host.

\section{METHODOLOGY}

\subsection{Picking out satellite candidates}

\footnotetext{
2 The reionization maps are available from the first author upon request.
}

Table 2

Properties of the reionization models

\begin{tabular}{cccrcc}
\hline \hline Model No. & $\left\langle z_{\text {reion }}\right\rangle$ & $\sigma_{z}$ & $z_{\text {end }}$ & Median & $\tau_{e}$ \\
\hline 1 & 9.31 & 1.44 & 7.44 & 8.84 & 0.075 \\
2 & 10.71 & 1.35 & 8.92 & 10.27 & 0.091 \\
3 & 11.97 & 1.28 & 10.27 & 11.57 & 0.108 \\
4 & 13.50 & 1.20 & 11.88 & 13.14 & 0.129 \\
\hline
\end{tabular}

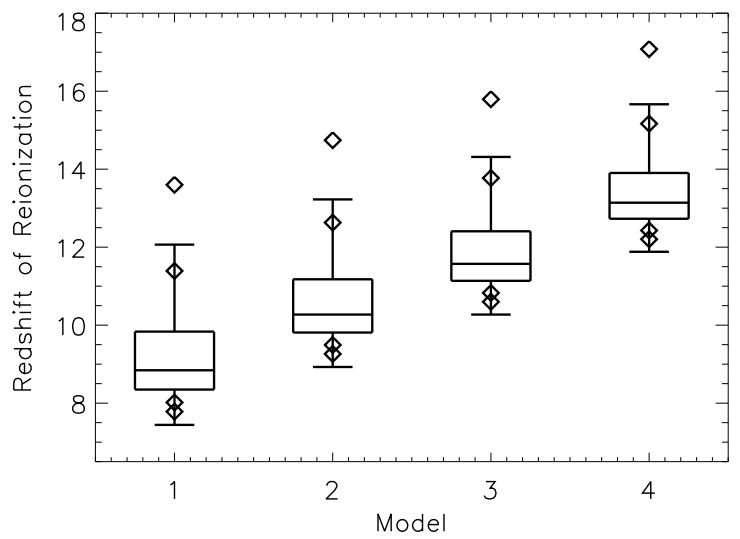

Figure 2. Boxplot summarizing the distributions of reionization redshifts over the cells in the $100 h^{-1} \mathrm{Mpc}$ box, for the four different models we considered. The boxes enclose the interquartile range, and the horizontal line is the median in each case. The whiskers extend to the highest and lowest value still within $1.5 \times$ the interquartile range, and the $2 \mathrm{nd}, 9$ th, 91 st and 98 th percentile are marked by diamonds.

We use the level-2 merger trees of the six Aquarius halos to determine which dark matter halos can host luminous satellite galaxies based on several criteria. These again reflect our assumptions about when a subhalo is able to accrete gas from the IGM, cool its gas, and form stars, following methods developed in e.g. Madau et al. (2008); Koposov et al. (2009); Busha et al. (2010). We only consider subhalos that end up within the virial radius of their host halos (see Table 11).

First, we require that subhalos must grow larger than a minimum $v_{\max }$ of $15 \mathrm{~km} / \mathrm{s}$ before star formation can take place, and also that this happens before the subhalo is reionized. This corresponds roughly to the virial temperature where atomic hydrogen is no longer able to cool $\left(\sim 10^{4} \mathrm{~K}\right)$. We do not explicitly include $\mathrm{H}_{2}$-cooling "minihalos" as sites for low-mass star formation before reionization, but recognize that such halos may have been the sites of the very first star formation, whose metals seeded the next generation of lower-mass, longlived stars (Bromm et al. 1999, 2003; Yoshida et al.|2004; Bromm et al. 2009).

Once a subhalo enters a reionized region (as described in Section 2.2), the minimum $v_{\max }$ needed to sustain star formation increases, reflecting both the increased temperature of the IGM to a few times $10^{4} \mathrm{~K}$ due to photoionization, and the possibility of photoevaporation of gas out of small halos (Barkana \& Loeb 1999; Iliev et al. 2005). Gnedin (2000) found the filtering mass to correspond to the scale at which the gas fraction in halos is significantly reduced due to reionization. Gnedin's 
expression, however, does not take into account that only the cold gas remains available for star formation; Muñoz et al. (2009) and Busha et al. (2010) argue that only halos that grow to a viral temperature $\sim 10^{5} \mathrm{~K}$ will be able to sustain star formation after reionization. Accordingly, we only let star formation continue post-reionization in subhalos whose $v_{\max }$ is greater than $50 \mathrm{~km} \mathrm{~s}^{-1}$. This represents a rather abrupt reionization effect; we also run a model where this suppression is weaker, with the threshold set at $30 \mathrm{~km} \mathrm{~s}^{-1}$.

In addition, we assume that once a satellite starts interacting with the main halo, its gas is stripped and no further star formation takes place. Thus, there are two important redshifts associated with each satellite: $z_{r e}$ and $z_{\text {infall }}$, the times when it is reionized and falls into the main halo, respectively.

In order to account for tidal stripping of satellites, which has been proposed as another important mechanism to resolve the missing satellite problem (Kravtsov et al. 2004), we tag the 1\% most bound particles of the subhalos as star-carrying when they are accreted onto the main halo. We then track all these tagged particles to redshift zero, and determine the final luminosity of a satellite based on its remaining tagged particles. In this way, we are able to take into account tidal stripping of satellites and other dynamical effects, under the assumption that the stars are found in the bottom of the potential well.

Of course, a number of infalling systems will be completely disrupted, and contribute to the accreted stellar halo. In Fig. 3. we show the projected distribution of all the dark matter particles that were tagged as stars on infall, in reionization scenario 1. Surviving satellites here appear as bright, concentrated dots, while the particles from the shredded systems make up the diffuse halo. Since the accreted stellar halos and streams forming in the Aquarius simulations have been studied extensively in Cooper et al. (2010) and Helmi et al. (2011), we will not discuss them further here. We note, however, that although our model is very simple, it still reproduces most of the features seen in Fig. 6 of Cooper et al. (2010) we take this as justification that our simple model still reasonably captures the relevant processes.

We determine satellite candidates for all four of our reionization calculations. In addition, we do the same for four "instantaneous" redshift models, where we set the reionization redshift equal to the mean reionization redshift in the entire box. Alvarez et al. (2009) showed that the distribution of reionization redshifts for Milky Way-sized halos is nearly similar to the global spatial distribution, making the global mean an appropriate choice. Comparing the resulting satellite populations from the patchy and instantaneous reionization models then allows us to isolate any effects of reionization not being spatially uniform.

\subsection{Assigning luminosities to halos}

In order to compare our satellite candidates to the observed Milky Way population, we need to assign luminosities to the subhalos. There are many different procedures for doing this; see e.g., Koposov et al. (2009) for a discussion. Here, we adopt a simple model where the star formation rate is proportional to the mass, that is
$M_{\text {star }}=\int \epsilon \times f_{\text {gas }} \times M_{D M} d t$, where $\epsilon=1 / \tau$ is a constant that sets the star formation efficiency (i.e., $\tau$ can be considered a timescale for which a subhalo would convert all of its gas into stars). We take $f_{\text {gas }} \times \epsilon=0.08 \times 10^{-10} \mathrm{yr}^{-1}$, tuned both so that we get a reasonable satellite population, but also so that the mass of the accreted stellar halo (i.e., the total mass of satellites that fall in and are completely shredded) falls in a mass range around $\sim 10^{9} M_{\odot}$ (Bell et al. 2008; Tumlinson 2010). This second criterion is insensitive to the reionization prescription, because the mass of the stellar halos is dominated by a few significant progenitors, while the smaller systems affected by reionization contribute a small fraction of the total mass. While in principle we could increase this product to get a better fit for the more extreme reionization scenarios, this would then lead to very massive stellar halos.

Our model is deliberately simple because we wish to assess and compare the impact of different reionization assumptions without getting addressing uncertainties in the baryonic physics. While we do not explicitly model effects like the impact of supernova feedback on the satellites, we assume that the typical effect is captured by setting $\epsilon$ (i.e., the star formation efficiency) appropriately. (For an example of a more extensive approach in modeling the baryonic content of dark halos in cosmological simulations, we refer the reader to Font et al. 2011.)

Having determined the stellar mass formed, we then assign each stellar population an associated presentday luminosity, using the stellar population synthesis (SPS) models of Bruzual \& Charlot (2003), assuming a Salpeter initial mass function, and a metallicity of $Z=0.0001$ (the lowest considered in their model, and which captures the typical range of the ultra-faint satellites we are most interested in, e.g., Kirby et al. 2008; Simon et al. 2010; Norris et al. 2010). We then track the particles tagged with stellar populations to redshift zero, and so determine the present-day luminosities of the satellite candidates picked out as described earlier.

As an alternative to the simple parametric method described above, we also try the abundance matching technique developed by Busha et al. (2010) to assign luminosities to dark matter halos. This is an extrapolation of the fit of Blanton et al. (2005) based on galaxies in the SDSS (down to $M_{r}=-12.375$ ) to fainter magnitudes, by a power law:

$$
M_{V}-5 \log h=18.2-2.5 \log \left[\left(\frac{v_{\max }}{1 \mathrm{~km} \mathrm{~s}^{-1}}\right)^{7.1}\right] .
$$

The appeal of the abundance matching procedure is that there are no parameters to tune, using a model derived from statistics of observed galaxies. While it is an extrapolation at UFD luminosities, Busha et al. (2010) showed that when applied to the Via Lactea subhalos, the abundance matching model did well in reproducing the observed luminosity function (but see Boylan-Kolchin et al. $2011 b$ for a discussion of potential problems with abundance matching for the MW dwarfs). For purposes of comparison, then, we also separately assign magnitudes to our subhalos using eqn. 11. In order to select an appropriate $v_{\max }$ for the calculation, we use the peak $v_{\max }$ of the subhalos during the time they were able to form stars, as defined in Section 3.1. For most subhalos, this 

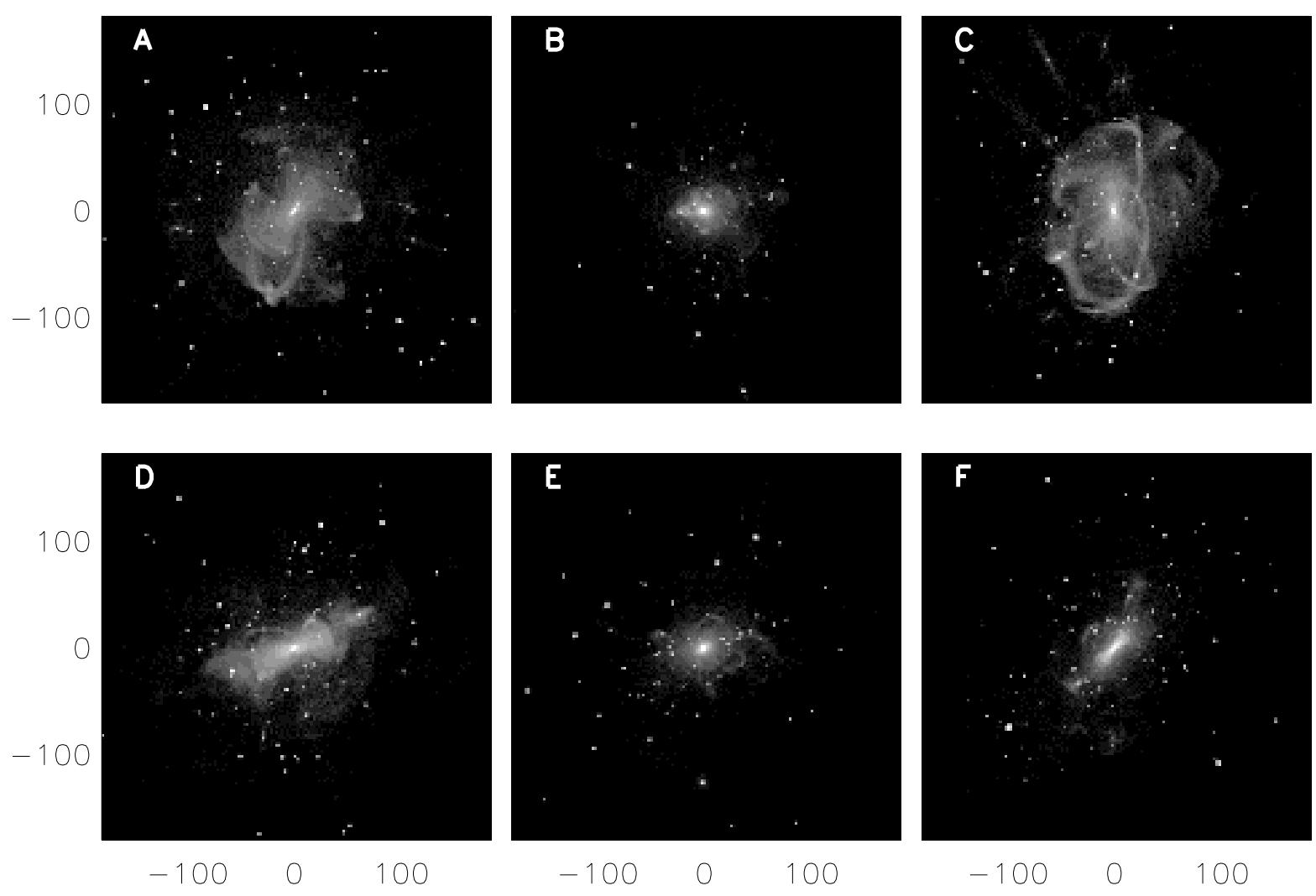

$-100 \quad 0$

100

Figure 3. Projected distribution of all dark matter particles that are tagged with stellar populations from infalling subhalos, at $z=0$. The scale is in kiloparsecs. Both the concentrated, bright satellites and the shredded satellites that make up the diffuse accreted stellar halo and streams are visible.

either corresponds to the time of reionization or time of accretion.

As some basic tests of our model, we check how well it reproduces various features of the observed Milky Way dwarf population, such as their radial distribution, and observed mass-to-light ratios. Fig. 4 shows the radial distribution of satellites in our simulation, as a fraction of total number. We find all six halos to have a satellite distribution consistent with what is observed around the Milky Way, with the possible exception of the B halo, which has a larger fraction of satellites somewhat closer in.

As for mass-to-light ratios, we note that subhalo masses in a simulation cannot be directly compared to observed dwarf galaxy masses. However, Walker et al. $(2009,2010)$ and Wolf et al. (2010) show that the mass within the half-light radius is well constrained for the Milky Way dwarfs. We thus estimate the half-light radius of our satellites from their assigned luminosities by fitting a power law to the data tabulated in Walker et al. (2010), and measure the mass within the half-light radius by fitting an NFW profile (Navarro et al. 1997). The result is shown in Fig. [5: the red points are simulated satellites, and the black crosses (UFDs) and triangles (classical dSphs) show the data from the Milky Way dwarf

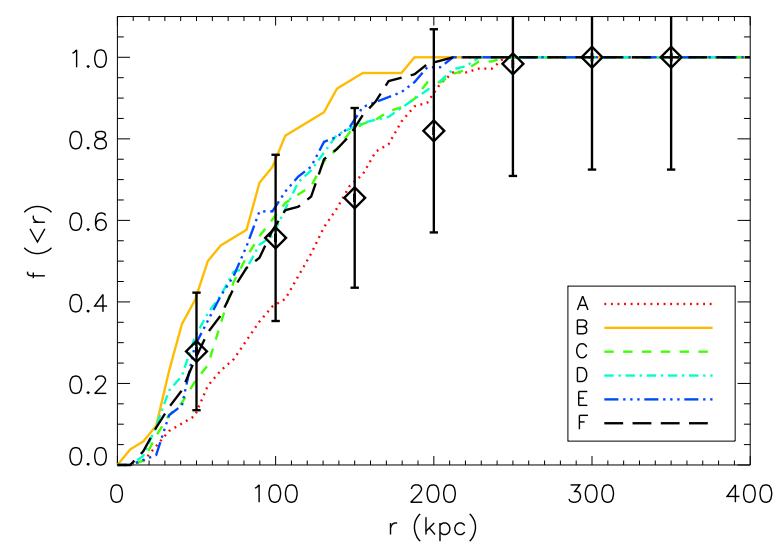

Figure 4. Radial distribution of satellites, as a fraction of total number. The points with error bars show the observed Milky Way distribution, where non-SDSS satellites are weighted by a factor of $f_{D R 5}=0.194$ of the total to account for incomplete sky coverage. The theoretical estimates are for reionization Model 1 with strong suppression, but the trends are similar for the other models. In particular, all halos have a radial satellite distribution consistent with that observed around the Milky Way, with the possible exception of the B halo, whose satellites are somewhat closer to the main halo. 
Table 3

Reionization times of the main halos in the four models

\begin{tabular}{cccccccc}
\hline \hline Model No. & $\mathrm{A}$ & $\mathrm{B}$ & $\mathrm{C}$ & $\mathrm{D}$ & $\mathrm{E}$ & $\mathrm{F}$ & Box Mean \\
\hline 1 & 9.2 & 9.9 & 12.0 & 9.3 & 8.5 & 8.1 & 9.31 \\
2 & 11.5 & 11.2 & 13.7 & 10.7 & 10.0 & 9.5 & 10.71 \\
3 & 12.6 & 12.4 & 16.3 & 12.0 & 11.8 & 10.9 & 11.97 \\
4 & 14.0 & 14.3 & 17.1 & 13.5 & 13.6 & 12.5 & 13.50 \\
\hline
\end{tabular}

galaxies. Our satellite properties are in good agreement with the observed values, and recover the observed relation between $M$ and $r_{0.5}$. The points shown are for satellites with the Model 1 reionization history; driving reionization to earlier times yield the same relation, but fewer satellites overall, and fewer in the intermediate $r_{0.5}$ (or luminosity) range. We conclude that our simple model is overall sufficient for reproducing the observed trends.

\section{RESULTS}

\subsection{Spread in reionization redshifts}

A major novel factor in our work is that the reionization redshift of a halo is completely determined by its environment. This has two main consequences: first, that the six main halos will reionize at different times according to their locations in the Millennium-II box. But additionally, the subhalos of each of these main halos are not required to reionize at the same time as their main halo - their reionization times are also set by their particular path through the main box. Here, we explore the distribution in reionization redshifts for the subhalos, and how this influences the resulting number of potential satellites.

Table 3 summarizes the first effect - that the six Aquarius host halos do not reionize at the same times in the box. In particular, the $\mathrm{F}$ halo consistently reionizes later than the box mean, while the $\mathrm{C}$ halo reionizes significantly earlier. The other four halos are closer to the mean of the box (and thus slightly earlier than the median; also see Table 2).

As for the second effect: Fig. 6] shows the distribution of satellite reionization redshifts for the six halos, here in the case of scenario 1 . (The distributions for the other three models show similar characteristics, but with fewer satellites and narrowing distributions as the models become more efficient.) Several interesting trends are evident: First, there is a distribution - while some of them are strongly peaked (C, D and F in particular), all show a range of redshifts. Second, the peak of the distribution does not necessarily correspond to the reionization redshift of their host halo (the dotted lines).

The $\mathrm{C}$ halo is the most extreme in this regard - the peak of the satellite distribution is near the mean of the box, while the host reionized significantly earlier. Essentially, this happens because the location of the $\mathrm{C}$ halo is near the edge of one of these early-reionized regions in the large box (cyan in Fig. 1), responsible for the early reionization of the $\mathrm{C}$ halo itself. Many of its satellites, however, fall in from a different direction than the bubble front, and end up with a reionization distribution peaking around the box mean. Note that Fig. 6] shows only systems that survive as self-bound satellites. If we were to also include systems that are shredded and are part of the diffuse main halo by redshift zero, we would see a larger number of subhalos reionizing around the same time as the $\mathrm{C}$ main halo.

Having established that there is indeed a distribution of satellite reionization redshifts, the next question is whether this leads to a different satellite population between patchy and instantaneous models. Fig. 7 considers the number of satellites produced in each model for different sets of assumptions: either patchy or instantaneous, and a suppression $v_{\max }$ of either 30 or $50 \mathrm{~km} / \mathrm{s}$. We see that regardless of cutoffs and patchy vs. instantaneous, as reionization is pushed to earlier times, there is a strong decline in the number of satellites. This overall trend is similar to what was found in previous studies (Muñoz et al. 2009; Tumlinson 2010; Busha et al. 2010). For a given reionization model, we do see additional differences, however. For either choice of suppression $v_{\max }$, the instantaneous reionization models systematically make 10-20\% more satellites than the corresponding patchy models; in the more extended models this effect dominates the difference between stronger and weaker suppression. As the reionization sources become more efficient and reionization occurs earlier, the patchiness is less important, and the variation in suppression $v_{\max }$ becomes the dominant effect in this scenario.

Note that the numbers shown in Fig. 7 are averages; as shown in Fig. 6] there is a substantial spread between the actual number of satellites between the different halos. The error bar indicates the typical $\mathrm{rms}, \pm \sim 15$ satellites. However, halos A-E all show more satellite candidates in the instantaneous scenarios, while the $\mathrm{F}$ halo has about $10 \%$ fewer. For five out of the six halos, then, the main effect of patchy reionization is that the number of satellite candidates is reduced by $10-20 \%$ compared to the instantaneous models. This can be understood in the context of Fig. 6, where the F halo is the only halo whose majority of satellites reionize later than the mean.

In practice, the difference between the patchy and instantaneous case in Fig. [7] is due to a combination of two effects: that the global mean is not necessarily the best instantaneous redshift to choose for a given halo, as well as not capturing the spread in reionization times. However, we do not find that using e.g. the host halo reionization redshift, or the median satellite reionization redshift, leads to better agreement in each case. While we could recover the number of satellites in each case by tuning the instantaneous reionization redshift, the value of such an exercise is limited: the only way to determine that appropriate redshift would be to already know the result from the patchy model. As such, this illustrates the main problem with using instantaneous and/or spatially homogeneous reionization models. Depending on the application, the fact that there is a distribution of satellite reionization redshifts may be important to the results - and this distribution cannot be captured in a homogeneous model, nor can the appropriate instantaneous approximation be recovered without knowing the patchy result.

\subsection{Luminosity Functions}

Having explored the reionization distributions in our simulations, we here discuss the resulting luminosity function of our simulated satellites. In order to compare them to the population of observed dwarfs, how- 


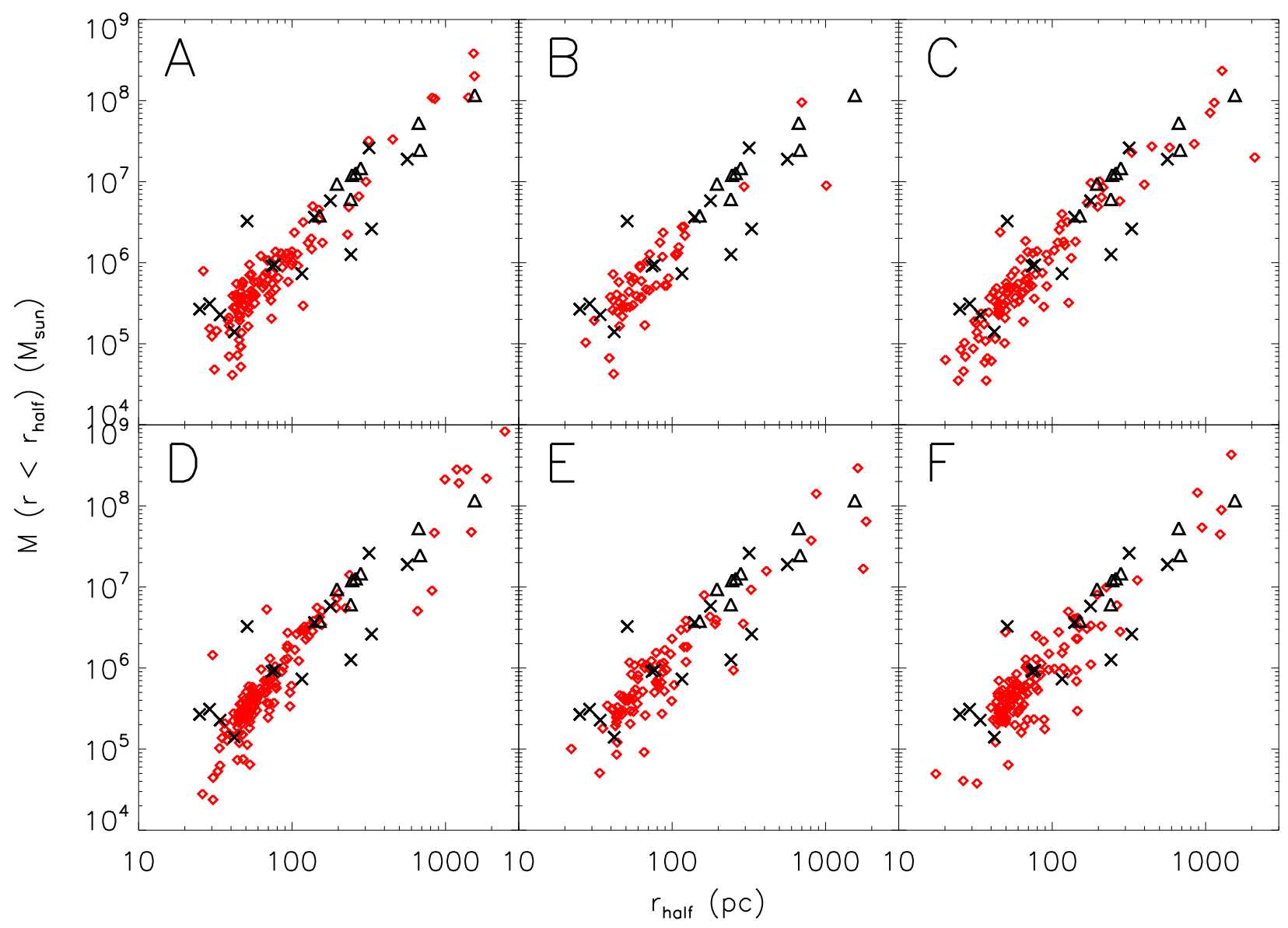

Figure 5. Mass within the estimated half-light radius versus half-light radius for the simulated satellites (red diamonds) for the six halos, in reionization scenario 1 , with the higher suppression $\left(v_{\max }=50 \mathrm{~km} \mathrm{~s}^{-1}\right)$. For the simulation points, the half-light radius is estimated from the luminosity via an empirical relation, and the mass is measured by fitting a NFW profile. Black triangles show the observed values in the Milky Way classical dSphs, while the crosses show the values for the UFDs.

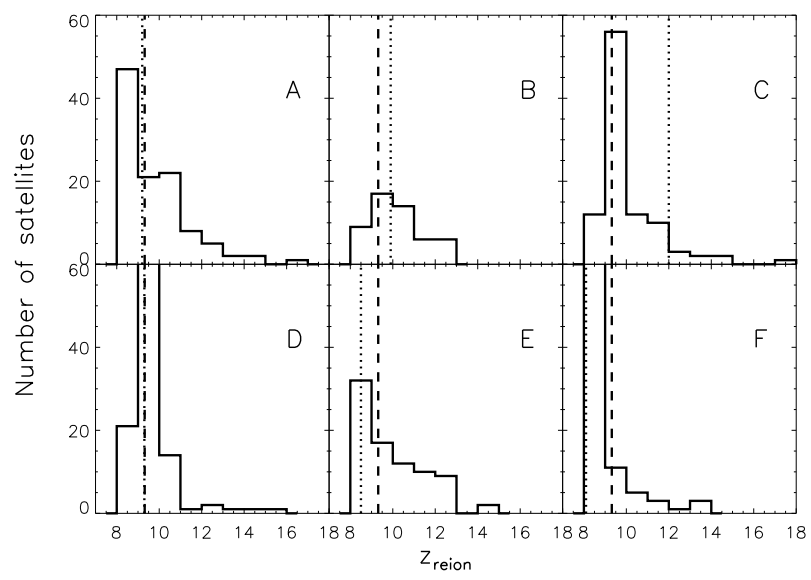

Figure 6. Distributions of reionization redshifts of the satellite candidates of the six halos, here shown for Model 1 and the higher $\left(v_{\max }=50 \mathrm{~km} \mathrm{~s}^{-1}\right)$ suppression threshold. The dashed lines correspond to the mean reionization redshift across the entire box in Model 1, while the dotted lines show the reionization redshifts of the six main halos. Note the tails to higher redshifts. ever, we first need to correct the observational sample of dwarf galaxies (data taken from Martin et al. 2008; Wadepuhl \& Springel 2011) for incompleteness and biases to establish a luminosity function. We follow the method outlined in Koposov et al. (2008) and Tollerud et al. (2008), which employed models for the completeness of the SDSS Data Release 5 (DR5) to quantify the detection efficiency and thus adjust the observed luminosity function. In addition, we only include the simulated satellites that would be detectable according to the DR5 criteria:

$$
r_{\max }=\left(\frac{3}{4 \pi f_{D R 5}}\right)^{1 / 3} 10^{\left(-0.6 M_{V}-5.23\right) / 3} \mathrm{Mpc},
$$

where $f_{D R 5}=0.194$ is the fraction of the sky covered by DR5. That is, simulated satellites with $r>r_{\max }\left(M_{V}\right)$ are not included in the plots in this section. In each case, $r_{\max }$ is calculated with respect to a point $8 \mathrm{kpc}$ from the center of the main halo.

Figure 8 shows the resulting cumulative luminosity function for the simulated satellite population of all six halos, for the four patchy reionization models and with $v_{\max }=50 \mathrm{~km} \mathrm{~s}^{-1}$. The luminosities shown are those obtained with the abundance matching technique (eqn. 1), 


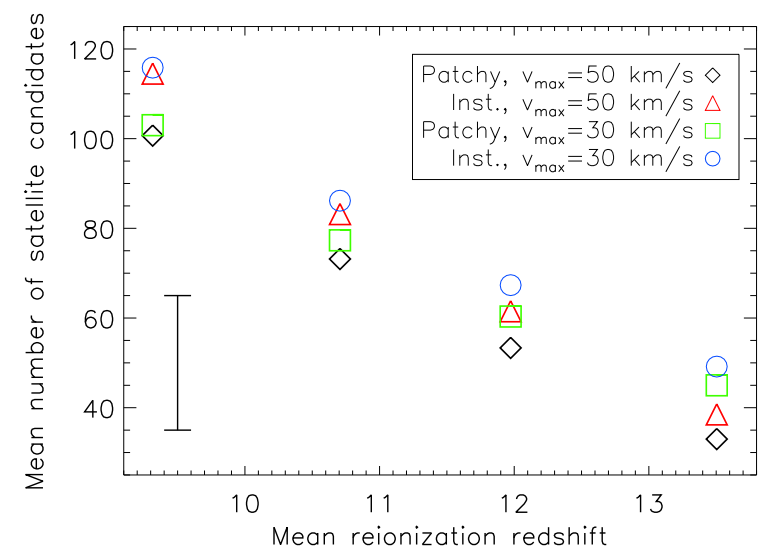

Figure 7. Mean number of satellite candidates for the six halos, for our four different reionization models, with varying assumptions: Either strong suppression at reionization, affecting all halos with $v_{\max }<50 \mathrm{~km} \mathrm{~s}^{-1}$, or a weaker suppression at $30 \mathrm{~km} \mathrm{~s}^{-1}$. In addition to the patchy cases, we also show calculations where reionization instead happened instantaneously at the mean redshift of each patchy model. While the trends with reionization model and suppression $v_{\max }$ are similar for the six halos, there is substantial halo-halo scatter in the actual number of satellite candidates. This is indicated by the error bar (typical rms $\sim 15$ satellites).

but the resulting luminosity functions are very similar to those using our simple SPS-based model. (However, there is substantial scatter between the methods on a subhalo-subhalo basis, i.e., rms $\sim 1$ mag.) The dotted line shows the resulting luminosity function when applying the abundance matching prescription to all subhalos in each simulation that lie within the virial radius by redshift $z=0$, without assuming any suppression due to baryonic processes. As such, it gives a good illustration of the missing satellite problem: without some process such as reionization to suppress star formation and reduce the number of luminous satellites, the six Aquarius halos would predict between 500-1000 satellites around Milky Way-like galaxies.

We see that while satellites brighter than $M_{V} \sim-10$ are insensitive to the reionization model, the total number and the number of faint satellites change dramatically with more efficient reionization. This is consistent with the results of Font et al. (2011), who found that photoheating is mainly important for suppressing star formation in satellites analogous to ultra-faint dwarfs. In our simulations, only Model 1 , and in some cases Model 2 , can match the faint end of the luminosity function. In fact, there is a factor of 3-4 difference between the total number of satellites predicted between the different reionization models for a given halo. This behavior may ultimately allow for constraining the Milky Way reionization epoch with the faintest satellites.

Another striking feature of Fig. 8 is the scatter between the six halos - there is a factor of $\sim 3$ difference between the $\mathrm{B}$ and $\mathrm{D}$ halos, which have the fewest and most satellites, respectively. Thus, the halo-halo scatter is of the same order as the differences in the impact of the various reionization models on the faint end. In order to break this degeneracy, it will be necessary to understand the general distribution of substructure around Milky Way-like halos, requiring a statistical sample of halos at sufficient resolution. Since the bright end of the luminosity function is not affected by the reionization history, however, it may be possible to break the degeneracy by constraining the halo-halo scatter using the brighter satellites, and use the fainter satellites to constrain the reionization epoch of the Milky Way.

We find that only the $\mathrm{F}$ halo hosts a satellite as bright as the Magellanic Clouds. This is consistent, however, with recent studies based on SDSS DR7 on the abundance of close, bright satellites around Milky Way-like galaxies (Liu et al. 2011; Bovlan-Kolchin et al. 2011a; Busha et al. 2011; Guo et al. 2011; Tollerud et al.|2011), and the possibility that the Clouds have entered the Milky Way halo only recently (Besla et al. 2007, 2010). Hence, we do not consider the mismatch at the brightest magnitudes to be problematic, especially given the simplicity of our star formation model.

Similar to the brighter satellites, the total stellar masses in the accreted halo of shredded, infalling satellites do not change significantly with reionization model. This is consistent with the results of Cooper et al. (2010), who studied in detail the building of accreted stellar halos in the Aquarius simulations. They found that the significant progenitors are similar in mass to the brightest Milky Way dwarf spheroidals, with each halo assembled from less than five such objects.

To highlight some of the differences between the various model assumptions, Fig. 9] shows the luminosity function of the $\mathrm{D}$ halo satellites for some parameter choices. The upper panels show the results using patchy reionization and $v_{\max }=50 \mathrm{~km} \mathrm{~s}^{-1}$, instantaneous reionization and $v_{\max }=50 \mathrm{~km} \mathrm{~s}^{-1}$, and patchy reionization and $v_{\max }=30 \mathrm{~km} \mathrm{~s}^{-1}$ respectively. The three lower panels show the same, but with luminosities determined by abundance matching (eqn. 11).

While the models with post-reionization star formation suppressed at $v_{\max }=50 \mathrm{~km} \mathrm{~s}^{-1}$ give a good match to the observed luminosity function, when we decrease the suppression threshold to $30 \mathrm{~km} \mathrm{~s}^{-1}$ none of the models fit the bias-corrected luminosity function well, despite the overall number of satellites only being slightly increased. This holds true both for the abundance matching-derived luminosities, and the best-fit SFR/SPS model. In particular, even if the star formation efficiency is decreased to give the correct number of satellites overall, the shape still does not match the observed luminosity function. It is possible, however, that this lower suppression model could still be a good match, if we explicitly consider other processes like supernova feedback (Li et al. 2010).

Compared to patchy reionization, the instantaneous models generally yield more satellites. While only the $\mathrm{D}$ halo is shown here as an illustration, as discussed in Section 4.1 this holds true for all of the Aquarius halos, except for the $\mathrm{F}$ halo. It is interesting to note, however, that the differences between different reionization models, luminosity prescriptions, etc., are in general dominated by the halo-halo scatter. That is, the differences in the satellite populations of the six halos for any given model are as large as the differences between the range of models we are exploring. As an example, Figure 10 shows the satellite luminosity functions for all six halos overplotted, here for reionization model 1 and the SFR luminosity prescription. While the $\mathrm{B}$ halo, which is the 


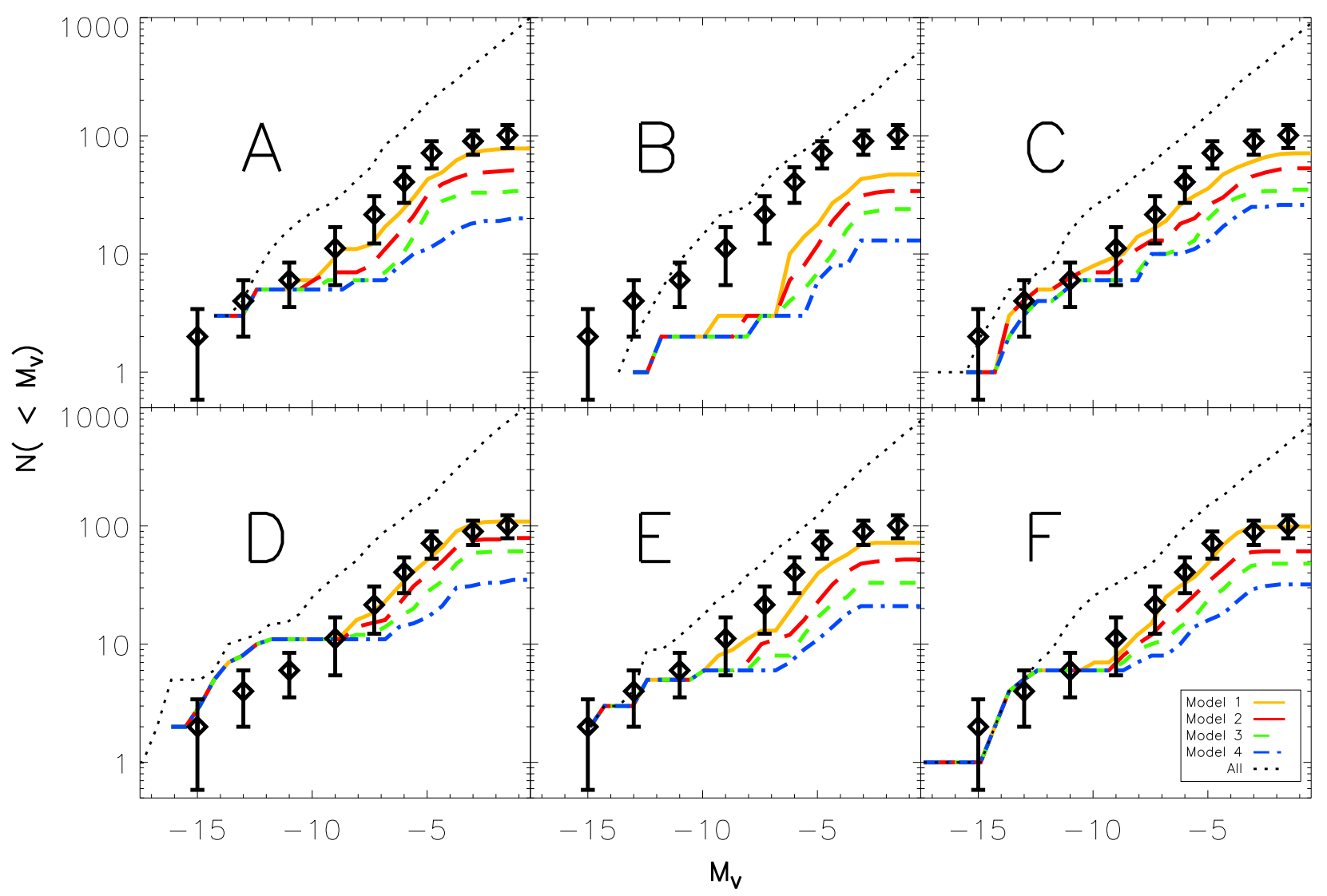

Figure 8. Cumulative luminosity functions of the simulated satellite populations of the six halos, using the abundance matching technique to assign luminosities. The colored curves show the results for the four patchy reionization models, with strong suppression at reionization $\left(v_{\max }=50 \mathrm{~km} \mathrm{~s}^{-1}\right)$. The black points with error bars show the observed Milky Way satellite luminosity function, applying the completeness correction of Koposov et al. (2008) and Tollerud et al. (2008). The dotted line shows what the luminosity function for all the subhalos would be if we ignored all baryonic effects, and used the same abundance matching extrapolation to assign luminosities to all subhalos in each case.

clear outlier, is also the lowest mass halo in this simulation suite, the uncertainty of where the Milky Way itself fits in this distribution makes direct comparisons to constrain models difficult.

\section{DISCUSSION AND CONCLUSIONS}

In our models, we find that the population of faint satellites is highly sensitive to the reionization model. This agrees with the results of Busha et al. (2010); Font et al. (2011), but is at odds with several earlier studies in the literature (Somerville 2002; Kravtsov et al. 2004). The difference is largely due to two factors. First, the previous work that focused on the range of the classical dwarf galaxies of the Milky Way found little reionization dependence; our results agree to the extent that the bright end of our luminosity function (classical dSph regime with $10^{5}<L<10^{8} L_{\odot}$ ) does not change either with large scale (different reionization model) or small scale (patchy versus instantaneous) changes in reionization. The difference at the faint end is explained by the various assumptions about how reionization influences the available supply of cold gas in small halos - our model assumes an abrupt end to star formation in halos smaller than a cutoff $v_{\max }$, whereas the filtering mass formalism will lead to a gradual suppression in star formation, and a much weaker reionization dependence. In that sense, we have chosen a maximum effect, and the results of our study can be viewed as an upper limit to the impact of patchiness of reionization.

We also note that since all but the largest subhalos in our simulation finish their star formation around reionization, our results are consistent with some ultrafaint dwarf galaxies having formed around the epoch of reionization in atomic cooling halos (but see e.g., Bovill \& Ricotti 2009). Given the cutoff we set at the atomic cooling threshold, our model does not predict objects much fainter than the faintest dwarf galaxies already observed, although this could potentially change if $\mathrm{H}_{2}$ cooling at high redshift were included.

Our main conclusions can then be summarized as follows:

- Patchy reionization models yield a distribution in satellite reionization redshifts, which leads to a 10$20 \%$ difference in the number of satellites they predict compared to instantaneous models. This effect 


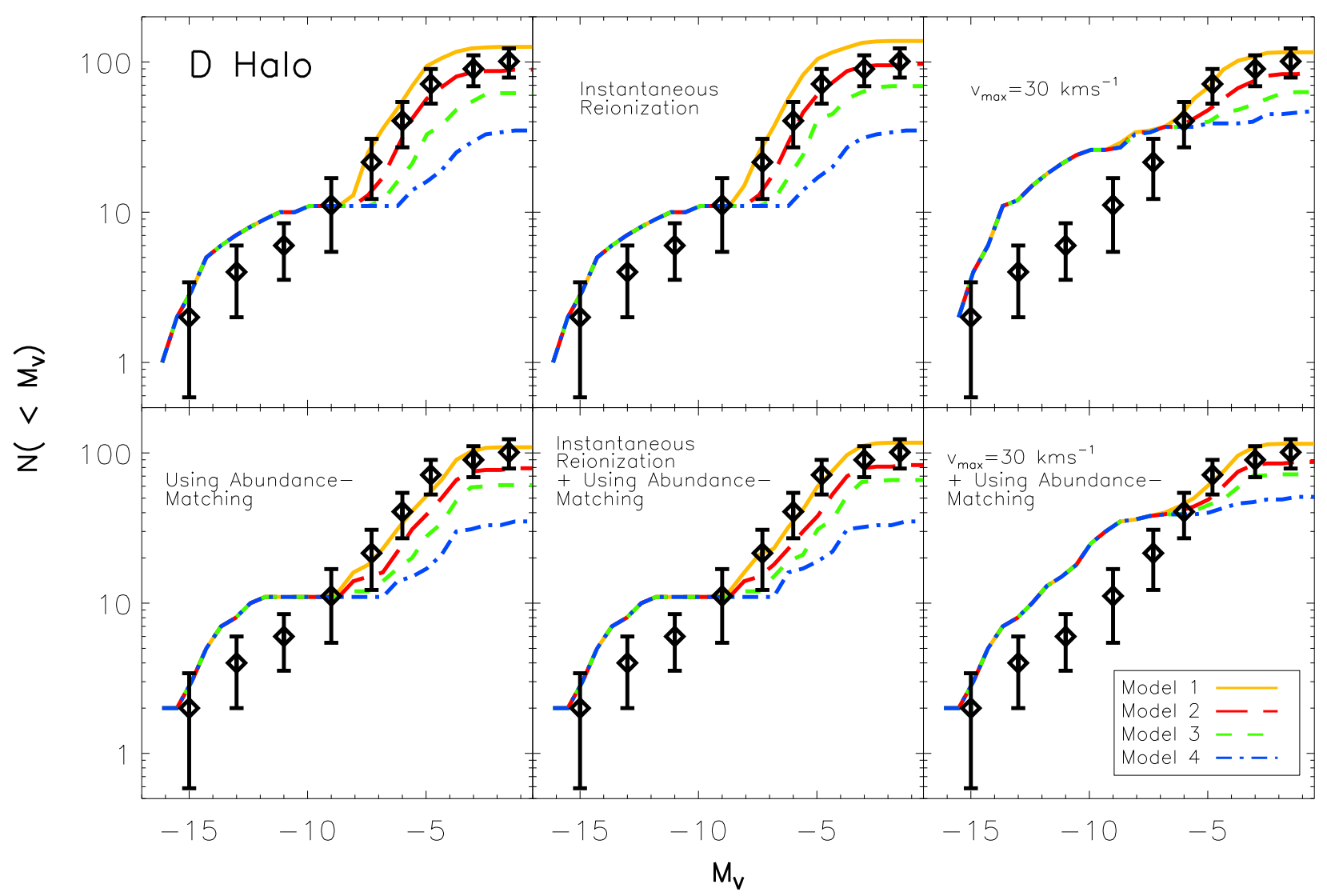

Figure 9. Comparisons of the different models, here illustrated by the resulting luminosity function for satellites of the D halo. Upper left: same as Fig. 8 the patchy reionization model, with luminosities assigned by the SPS method, for $v_{\max }=50 \mathrm{~km} \mathrm{~s}{ }^{-1}$. Upper middle: instantaneous reionization at the mean reionization redshift of the model. Upper right: patchy, with threshold for suppressing star formation lowered to $v_{\max }=30 \mathrm{~km} \mathrm{~s}^{-1}$. Lower panels: same as upper, but with luminosities assigned by the abundance matching technique, rather than the SPS method.

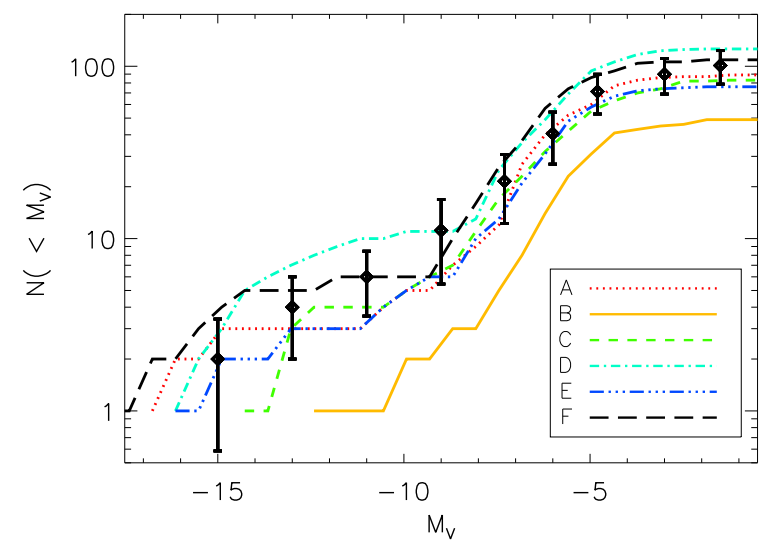

Figure 10. Illustrating halo-halo scatter: Overplotting the luminosity functions of the satellite populations of all the six halos, here shown for reionization model 1 (yellow line in Figs. 8 and 9).

can result in more or fewer satellites depending on the overall environment and merger history of the halo, and as such there is no general way to correct for it in a homogeneous model.

- The overall number of satellites depends sensitively on the reionization model - we find a factor of 3-4 difference between the earliest and latest reionization model. The difference is entirely in the number of fainter satellites, while the brighter end of the luminosity function corresponding to the classical $\mathrm{dSph}$ regime is unaffected.

- There are large halo-halo variations in the number of luminous satellites for a Milky Way sized halowe find a factor of 2-3 difference between the halo with fewest and most satellites, comparable to the spread between the different reionization models. Hence, in order to use the Milky Way satellites to constrain reionization, a much larger sample of halo simulations is required. Given the spread between the six halos in the Aquarius sample, the direct comparison of one or a few halos to the observed Milky Way will only yield conclusive results once we have a more detailed understanding of how our Galaxy fits into this distribution.

Progress can be made in several directions. On 
the observational side, future and ongoing surveys like SkyMapper, PanSTARRS and LSST (Keller et al. 2007; Kaiser et al. 2002; Ivezic et al. 2008) will cover the entire sky at the depth of SDSS or deeper, reducing the uncertainty in the number of observed dwarf galaxies due to the incomplete sky coverage of SDSS. Follow-up studies of new and existing dwarf galaxies can also provide more observational constraints, which can help discriminate between the different theoretical models that at present all fit the observed luminosity function.

On the simulation side, the present work has shown that in a six-halo sample, there is a large degeneracy between the significant halo-to-halo scatter and the results of effects like reionization. This level of cosmic variance will need to be quantified more accurately with a larger set of simulated halos. Only then can the origin and evolution of the faintest galaxies in the universe, as well as their role in building larger galaxies be more thoroughly understood.

\section{ACKNOWLEDGMENTS}

We thank the Virgo Consortium for access to the Aquarius simulations for this project, and Matt Walker and Daniel Eisenstein for helpful discussions. We also thank the anonymous referee for helpful comments that improved the structure of the manuscript. A.F. acknowledges support of a Clay Fellowship administered by the Smithsonian Astrophysical Observatory.

\section{REFERENCES}

Ahn, K., Shapiro, P. R., Iliev, I. T., Mellema, G., \& Pen, U. 2009, ApJ, 695, 1430

Alvarez, M. A., Busha, M., Abel, T., \& Wechsler, R. H. 2009, ApJL, 703, L167

Barkana, R., \& Loeb, A. 1999, ApJ, 523, 54

-. 2004, ApJ, 609, 474

Bell, E. F., et al. 2008, ApJ, 680, 295

Belokurov, V., et al. 2006, ApJ, 647, L111

Benson, A. J., Frenk, C. S., Lacey, C. G., Baugh, C. M., \& Cole, S. 2002a, MNRAS, 333, 177

Benson, A. J., Lacey, C. G., Baugh, C. M., Cole, S., \& Frenk, C. S. 2002b, MNRAS, 333, 156

Besla, G., Kallivayalil, N., Hernquist, L., Robertson, B., Cox, T. J., van der Marel, R. P., \& Alcock, C. 2007, ApJ, 668, 949

Besla, G., Kallivayalil, N., Hernquist, L., van der Marel, R. P. Cox, T. J., \& Kereš, D. 2010, ApJ, 721, L97

Blanton, M. R., Lupton, R. H., Schlegel, D. J., Strauss, M. A., Brinkmann, J., Fukugita, M., \& Loveday, J. 2005, ApJ, 631, 208

Bovill, M. S., \& Ricotti, M. 2009, ApJ, 693, 1859

Boylan-Kolchin, M., Besla, G., \& Hernquist, L. 2011a, MNRAS, 414, 1560

Boylan-Kolchin, M., Bullock, J. S., \& Kaplinghat, M. 2011b, MNRAS, 415, L40

Boylan-Kolchin, M., Springel, V., White, S. D. M., Jenkins, A., \& Lemson, G. 2009, MNRAS, 398, 1150

Bromm, V., Coppi, P. S., \& Larson, R. B. 1999, ApJ, 527, L5

Bromm, V., Yoshida, N., \& Hernquist, L. 2003, ApJ, 596, L135

Bromm, V., Yoshida, N., Hernquist, L., \& McKee, C. F. 2009, Nature, 459, 49

Bruzual, G., \& Charlot, S. 2003, MNRAS, 344, 1000

Busha, M. T., Alvarez, M. A., Wechsler, R. H., Abel, T., \& Strigari, L. E. 2010, ApJ, 710, 408

Busha, M. T., Wechsler, R. H., Behroozi, P. S., Gerke, B. F., Klypin, A. A., \& Primack, J. R. 2011, ApJ, 743, 117

Cooper, A. P., et al. 2010, MNRAS, 406, 744

D'Onghia, E., Besla, G., Cox, T. J., \& Hernquist, L. 2009, Nature, 460, 605

D’Onghia, E., Springel, V., Hernquist, L., \& Keres, D. 2010, ApJ, 709,1138
Efstathiou, G. 1992, MNRAS, 256, 43P

Font, A. S., et al. 2011, MNRAS, 417, 1260

Frebel, A., \& Bromm, V. 2010, ArXiv e-prints, 1010.1261

Frebel, A., Simon, J. D., Geha, M., \& Willman, B. 2010, ApJ, 708,560

Furlanetto, S. R., Zaldarriaga, M., \& Hernquist, L. 2004a, ApJ, 613,16

-. 2004b, ApJ, 613, 1

Gnedin, N. Y. 2000, ApJ, 542, 535

Guo, Q., Cole, S., Eke, V., \& Frenk, C. 2011, MNRAS, 417, 370

Helmi, A., Cooper, A. P., White, S. D. M., Cole, S., Frenk, C. S., \& Navarro, J. F. 2011, ApJ, 733, L7+

Iliev, I. T., Shapiro, P. R., \& Raga, A. C. 2005, MNRAS, 361, 405

Ivezic, Z., Tyson, J. A., Allsman, R., Andrew, J., Angel, R., \& for the LSST Collaboration. 2008, ArXiv e-prints, 0805.2366

Kaiser, N., et al. 2002, in Society of Photo-Optical

Instrumentation Engineers (SPIE) Conference Series, Vol. 4836,

Society of Photo-Optical Instrumentation Engineers (SPIE)

Conference Series, ed. J. A. Tyson \& S. Wolff, 154-164

Keller, S. C., et al. 2007, PASA, 24, 1

Kirby, E. N., Simon, J. D., Geha, M., Guhathakurta, P., \& Frebel, A. 2008, ApJL, 685, L43

Klypin, A., Kravtsov, A. V., Valenzuela, O., \& Prada, F. 1999, ApJ, 522, 82

Komatsu, E., et al. 2011, ApJS, 192, 18

Koposov, S., et al. 2008, ApJ, 686, 279

Koposov, S. E., Yoo, J., Rix, H., Weinberg, D. H., Macciò, A. V., \& Escudé, J. M. 2009, ApJ, 696, 2179

Kravtsov, A. V., Gnedin, O. Y., \& Klypin, A. A. 2004, ApJ, 609, 482

Li, Y., De Lucia, G., \& Helmi, A. 2010, MNRAS, 401, 2036

Lidz, A., McQuinn, M., Zaldarriaga, M., Hernquist, L., \& Dutta, S. 2007, ApJ, 670, 39

Liu, L., Gerke, B. F., Wechsler, R. H., Behroozi, P. S., \& Busha, M. T. 2011, ApJ, 733, 62

Macciò, A. V., Kang, X., Fontanot, F., Somerville, R. S., Koposov, S., \& Monaco, P. 2010, MNRAS, 402, 1995

Madau, P., Kuhlen, M., Diemand, J., Moore, B., Zemp, M., Potter, D., \& Stadel, J. 2008, ApJL, 689, L41

Martin, N. F., de Jong, J. T. A., \& Rix, H.-W. 2008, ApJ, 684, 1075

McQuinn, M., Lidz, A., Zahn, O., Dutta, S., Hernquist, L., \& Zaldarriaga, M. 2007, MNRAS, 377, 1043

Moore, B., Ghigna, S., Governato, F., Lake, G., Quinn, T., Stadel, J., \& Tozzi, P. 1999, ApJ, 524, L19

Muñoz, J. A., Madau, P., Loeb, A., \& Diemand, J. 2009, MNRAS, 400, 1593

Navarro, J. F., Frenk, C. S., \& White, S. D. M. 1997, ApJ, 490, 493

Norris, J. E., Wyse, R. F. G., Gilmore, G., Yong, D., Frebel, A., Wilkinson, M. I., Belokurov, V., \& Zucker, D. B. 2010, ApJ, 723,1632

Simon, J. D., Frebel, A., McWilliam, A., Kirby, E. N., \&

Thompson, I. B. 2010, ApJ, 716, 446

Simon, J. D., \& Geha, M. 2007, ApJ, 670, 313

Somerville, R. S. 2002, ApJ, 572, L23

Spergel, D. N., et al. 2007, ApJS, 170, 377

Springel, V., White, S. D. M., Tormen, G., \& Kauffmann, G. 2001, MNRAS, 328, 726

Springel, V., et al. 2005, Nature, 435, 629

-. 2008, MNRAS, 391, 1685

Tollerud, E. J., Boylan-Kolchin, M., Barton, E. J., Bullock, J. S., \& Trinh, C. Q. 2011, ApJ, 738, 102

Tollerud, E. J., Bullock, J. S., Strigari, L. E., \& Willman, B. 2008, ApJ, 688, 277

Tumlinson, J. 2010, ApJ, 708, 1398

Wadepuhl, M., \& Springel, V. 2011, MNRAS, 410, 1975

Walker, M. G., Mateo, M., Olszewski, E. W., Peñarrubia, J., Wyn Evans, N., \& Gilmore, G. 2009, ApJ, 704, 1274

-. 2010, ApJ, 710, 886

Willman, B., et al. 2005, AJ, 129, 2692

Wolf, J., Martinez, G. D., Bullock, J. S., Kaplinghat, M., Geha, M., Muñoz, R. R., Simon, J. D., \& Avedo, F. F. 2010, MNRAS, 406, 1220

Yoshida, N., Bromm, V., \& Hernquist, L. 2004, ApJ, 605, 579

Zahn, O., Lidz, A., McQuinn, M., Dutta, S., Hernquist, L., Zaldarriaga, M., \& Furlanetto, S. R. 2007, ApJ, 654, 12 
Zucker, D. B., et al. 2006, ApJ, 650, L41 